\title{
McKean-Vlasov type stochastic differential equations arising from the random vortex method
}

\author{
Zhongmin Qian ${ }^{1,2}$ (D) Yuhan Yao ${ }^{1}$
}

Received: 23 July 2021 / Accepted: 13 December 2021 / Published online: 24 December 2021

(c) The Author(s) 2021

\begin{abstract}
We study a class of McKean-Vlasov type stochastic differential equations (SDEs) which arise from the random vortex dynamics and other physics models. By introducing a new approach we resolve the existence and uniqueness of both the weak and strong solutions for the McKean-Vlasov stochastic differential equations whose coefficients are defined in terms of singular integral kernels such as the Biot-Savart kernel. These SDEs which involve the distributions of solutions are in general not Lipschitz continuous with respect to the usual distances on the space of distributions such as the Wasserstein distance. Therefore there is an obstacle in adapting the ordinary SDE method for the study of this class of SDEs, and the conventional methods seem not appropriate for dealing with such distributional SDEs which appear in applications such as fluid mechanics.
\end{abstract}

Keywords Aronson estimates · Cameron-Martin formula $\cdot$ Diffusion processes · McKean-Vlasov SDEs · Strong solution · Vorticity equation

Mathematics Subject Classification 60H30 · 35Q30 - 35Q35 · 76D03 · 76D05 · 76D17

This article is part of the section "Theory of PDEs" edited by Eduardo Teixeira.

Z. Qian: The datasets generated during and/or analysed during the current study are available from the corresponding author on reasonable request.

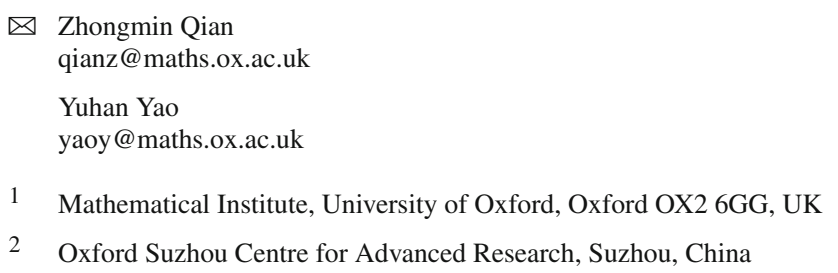




\section{Introduction}

In this paper, we study the following McKean-Vlasov type stochastic differential equations

$$
\left\{\begin{array}{l}
d X^{i}(x, t)=\left(\left.\sum_{j=1}^{d} \int_{\mathbb{R}^{d}} \mathbb{E}\left[K_{j}^{i}(z-X(y, t))\right]\right|_{z=X(x, t)} \omega_{0}^{j}(y) d y\right) d t+\sqrt{2 v} d B^{i}(t), \\
X(x, t)=x, \text { for } x \in \mathbb{R}^{d}
\end{array}\right.
$$

where $i=1, \ldots, d, v>0$ is a constant (which has its origin in fluid mechanics, namely the kinetic viscosity), $B=\left(B^{1}, \ldots, B^{d}\right)$ is a standard Brownian motion on some probability space and $\omega_{0}=\left(\omega_{0}^{1}, \ldots, \omega_{0}^{d}\right)$ is the initial data to the corresponding non-linear (and nonlocal) partial differential equations (PDEs), see (6.2) below. The structure kernel function which defines $\operatorname{SDE}(1.1) K=\left(K_{j}^{i}\right)$ is a $d \times d$ matrix-valued Borel measurable function on $\mathbb{R}^{d}$, which is continuous except at several singularities. The study of SDE (1.1) is inspired from the random vortex method in fluid mechanics, in which the integral kernel $K$ is singular at 0 . We will explain the random vortex model and formulate the problem (1.1) more precisely in the next section.

Equation (1.1) is a system of SDEs which involves the distributions of its solutions. This type of SDEs and SDEs which share the same nature may arise from physics models and from applied mathematics, and they have been studied intensively over the past decades. There is a large amount of literature devoting to various aspects of McKean-Vlasov equations, initiated by McKean in his seminal paper [21] (see for example [8,15,34] for some recent progress, $[5-7,22,29,35,38,40]$ and the literature therein).

The study of McKean-Valsov SDEs and the renewed interest in SDEs involving solution distributions in recent years are largely influenced by their connections with some nonlocal and non-linear PDEs arising from physics models. In this aspect, McKean-Valsov type SDEs provide the theoretical foundation for numerical methods such as the particle method for simulating the solutions to this kind of PDEs. For example, the propagation of chaos (law of large numbers) for solving the corresponding PDE of (1.1) may be formulated by replacing the expectation by the empirical measure, to obtain the following system

$$
\left\{\begin{array}{l}
d X^{n, \boldsymbol{k}}=\frac{1}{N} \sum_{n=1}^{N} \sum_{j} \varepsilon^{d} K\left(X^{n, \boldsymbol{k}}-X^{n, \boldsymbol{j}}\right) \omega^{j} d t+\sqrt{2 v} d B^{n}(t), \\
X^{n, \boldsymbol{k}}(0)=\varepsilon \boldsymbol{k}, \text { for } \boldsymbol{k} \in \mathbb{Z}^{d},
\end{array}\right.
$$

where $B^{n}$ are independent copies of $d$-dimensional Brownian motion, and $\varepsilon>0$ is the lattice size. The previous random system is the essential ingredient in the random vortex method, see for example $[20,22,25,26]$. Other numerical approximations have also been employed to look for large deviation results, see for example $[4,11,18,19]$ for detailed discussions. The difficulty however, in particular in the case that the dimension $d=3$ and $K$ is the BiotSavart kernel, comes from the fact that the kernel $K$ is too singular at 0 , hence the Lipschitz continuity of the coefficients appearing in (1.1), which is essential (see for example [8,34]), can not be expected.

SDE (1.1) has an independent interest by its own of course besides its significance in fluid dynamics. The research for this type of SDEs has been dominated, to the best knowledge of the present authors, by the use of Itô's SDE theory in one or another way which requires the Lipschitz continuity of $K$ with respect to the variational distance or the Wasserstein distance when one seeks for strong solutions, or by means of martingale problem for weak solutions. Unfortunately, these approaches are not appropriate for the study of (1.1) when $K$ is singular 
such as the Biot-Savart kernel $-\frac{1}{4 \pi} \frac{x}{|x|^{3}}$ (where $d=3$ ) which is exploded near zero like $1 /|x|^{2}$.

In the present paper, we overcome these difficulties by devising a new and powerful approach which allows us to establish the existence and uniqueness of strong and weak solutions of (1.1) under very weak conditions on the singular integral kernel $K$. In particular, our results apply to the the Biot-Savart kernel in any dimension, and also apply to the Green kernels (such as $\ln |x|$ in dimension $2,1 /|x|^{d-2}$ for $d>2$ ), the Riesz kernels $1 /|x|^{\gamma}$ where $\gamma \in[0, d)$ on $\mathbb{R}^{d}$ and many other singular integral kernels.

Our novel approach is based on the following key observation. If $K$ is singular, then the mapping

$$
(x, \mu) \rightarrow \sum_{j=1}^{d} \int_{\mathbb{R}^{d}} \mathbb{E}\left[K_{j}^{i}(x-\xi)\right] \omega_{0}^{j}(y) d y
$$

where $\xi$ has a distribution $\mu$, is unlikely Lipschitz continuous with respect to the variational or the Wasserstein metric on the space of distributions. However we recognise that the distributions of possible solutions to (1.1), even $K$ is singular, have much higher regularity. In fact, if $\left\{X(x, t): x \in \mathbb{R}^{d}, t \geq 0\right\}$ is a (weak) solution to (1.1) then

$$
b^{i}(x, t)=\sum_{j=1}^{d} \int_{\mathbb{R}^{d}} \mathbb{E}\left[K_{j}^{i}(x-X(y, t))\right] \omega_{0}^{j}(y) d y
$$

defines a vector field (although the vector field $b(x, t)$ is defined via the solution of the SDE), and $X(x, t)$ must be a weak solution to the diffusion process defined by ordinary SDE

$$
d X_{t}=b\left(X_{t}, t\right) d t+\sqrt{2 v} d B_{t} .
$$

Therefore the distribution of $X(x, t)$ can be represented by Cameron-Martin formula in terms of the Wiener measure and many results from diffusion processes thus can be brought in to the study of SDE (1.1). In this paper the sharp heat kernel estimates obtained in [27,28], together with other key estimates we are going to establish, allow to achieve the resolution of the problem 1.1 .

The paper is organised as the following. In Sect. 2, we recall the random vortex problem and derive the SDE (1.1) from the vorticity equation and formulate SDE (1.1) in a form which will be appropriate in the work frame of the present paper. In Sect.3, we collect a few facts about diffusion processes whose infinitesimal generators are elliptic operators of second order, and we prove several technical estimates which will be used to prove our main results. In Sect.4, we define a non-linear mapping associated with SDE (1.1) and prove it is a contractive mapping, then we show that (1.1) admits a unique weak solution. In Sect. 5 we show that a strong solution can be constructed, and show that the drift vector field (1.3) is Hölder continuous. Section 6 recovers solutions to the corresponding non-linear PDEs by using the solutions to (1.1), which can be considered as a probabilistic representation for this class of non-local and non-linear PDEs.

Convention on Notations. The following set of conventions are employed throughout the paper. Firstly Einstein's convention on summation on repeated indices through their ranges is assumed, unless otherwise specified. If $A$ is a vector or a vector field (in the space of dimension $d$ ) dependent on some parameters, then its components are labelled with upperscript indices so that $A=\left(A^{i}\right)=\left(A^{1}, \ldots, A^{d}\right)$. The same convention applies to coordinates too, so that $x=\left(x^{i}\right)=\left(x^{1}, \ldots, x^{d}\right)$. If $u$ is a vector field on $\mathbb{R}^{3}$ then $\nabla \times u$ denotes the curl of $u$ which is again a vector field on $\mathbb{R}^{3}$ with its component $\varepsilon^{i j k} \frac{\partial u^{k}}{\partial x^{j}}$. If $f$ is a function on $\mathbb{R}^{d}$, 
then $\|f\|_{p}$ or $\|f\|_{L^{p}\left(\mathbb{R}^{d}\right)}$ denotes the $L^{p}$-norm of $f$ with respect to the Lebesgue measure. Similarly, if $f(x, t)$ is a function on $\mathbb{R}^{d} \times[0, T]$ then $\|f\|_{L^{p}\left(\mathbb{R}^{d} \times[0, T]\right)}$ or, if no confusion is possible, $\|f\|_{p}$ denotes the $L^{p}$-norm with respect to the Lebesgue measure on the product space $\mathbb{R}^{d} \times[0, T]$.

\section{Random vortex method-from PDE to SDE}

Particle formulations for fluid flows have been studied as a tool for understanding fluid dynamics of turbulence. The underlying idea is simple, originally due to Taylor [36]. Instead of considering the velocity vector field $u(x, t)$ of the flow, one may study the dynamics of trajectories $X(x, t)$ of the fluid particles emitting from $x$ at the moment 0 , i.e. the dynamical equation

$$
\frac{d}{d t} X(x, t)=u(X(x, t), t), \quad X(x, 0)=x
$$

and reformulate the equation of motion of the vorticity $\omega=\nabla \times u$ into an evolution equation for $X(x, t)$. This approach works well for certain inviscid fluids.

For viscous incompressible fluid with constant viscosity $v>0$, a natural idea is to consider Brownian particles instead, i.e. $X(x, t)$ is modelled by the Taylor diffusion

$$
d X(x, t)=u(X(x, t), t) d t+\sqrt{2 v} d B_{t}, \quad X(x, 0)=x
$$

where $B$ is a standard Brownian motion, and rewrite the equation of vorticity motion in terms of the distribution of the Taylor diffusion. This approach is called the random vortex method, see for example [9,10,12,19,30,37] and etc. for a comprehensive account including the recent exciting progress. For incompressible fluid flows, $u(x, t)$ satisfies the Navier-Stokes equations

$$
\begin{aligned}
\frac{\partial}{\partial t} u+u \cdot \nabla u & =v \Delta u-\nabla p, \\
\nabla \cdot u & =0
\end{aligned}
$$

where $p(x, t)$ is a scalar function representing the pressure at $(x, t)$. If the fluid is constrained in a finite region, then certain boundary conditions must be identified, but for simplicity, we consider the case where the evolution of the fluid can take place without physical boundary and also without external force. In this case, the implicit boundary condition at infinity is applied: both $u(x, t)$ and $p(x, t)$ tend to zero sufficiently fast as $|x| \rightarrow \infty$. This is the model used in the homogeneous turbulence for example. The incompressible condition (2.3) allows to reformulate the first equation (2.2) in terms of the fluid vorticity $\omega=\nabla \times u$, which is a vector field in $\mathbb{R}^{3}$ with its components $\omega^{i}=\varepsilon^{i j k} \frac{\partial u^{k}}{\partial x^{j}}$, and the equation of vorticity motion is the following vorticity equation

$$
\frac{\partial}{\partial t} \omega+u \cdot \nabla \omega=v \Delta \omega+\omega \cdot \nabla u,
$$

where the velocity field $u$ can be recovered from the Laplace equation

$$
\Delta u=-\nabla \times \omega .
$$




\subsection{Taylor's diffusions}

In our approach, Taylor's diffusions will play a crucial rôle, so the goal of this part is not only for the propose of describing the vortex dynamics, but also for establishing a few notions and notations which will be used frequently throughout the paper. The vorticity equation may be written as

$$
\left(\frac{\partial}{\partial t}-L_{-u}\right) \omega=\omega \cdot \nabla u,
$$

where we have introduced the following notation: if $b(x, t)$ is a time-dependent vector field (here $t$ is the time variable), then

$$
L_{b(x, t)}=v \Delta+b(x, t) \cdot \nabla
$$

which is a differential operator of second order and is time inhomogeneous in general. This convention will be applied to any time dependent vector field $b(x, t)$ on $\mathbb{R}^{d}$ where $d$ is not necessary to be 3 . If no confusion may arise, the argument $(x, t)$ will be suppressed. $L_{b}$ is the infinitesimal generator of the Taylor diffusion describing the motion of Brownian particles $\left(X_{t}\right)_{t \geq 0}$, which can be defined by the Itô's stochastic differential equation

$$
d X_{t}=b\left(X_{t}, t\right) d t+\sqrt{2 v} d B_{t}
$$

where $(B)_{t \geq 0}$ is a standard Brownian motion in $\mathbb{R}^{d}$ on some probability space $(\Omega, \mathcal{F}, \mathbb{P})$. The transition probability density function of the $L_{b}$-diffusion is denoted by $p_{b}(\tau, x, t, y)$ for $t>\tau \geq 0$ and $x, y \in \mathbb{R}^{d}$ in the sense that

$$
\mathbb{P}\left[X_{t} \in d y \mid X_{\tau}=x\right]=p_{b}(\tau, x, t, y) d y .
$$

The formal adjoint operator of $L_{b}$ is given by

$$
L_{b}^{\star}=v \Delta-b \cdot \nabla-\nabla \cdot b=L_{-b}-\nabla \cdot b
$$

which is again a diffusion operator if and only if the vector field $b$ is divergence-free. In particular, if $b(\cdot, t)$ is solenoidal then $L_{b}^{\star}=L_{-b}$. The following lemma contains the facts about the elliptic operator $L_{b}$ which will be used throughout the paper.

Lemma 1 Let $b(x, t)$ be a time-dependent Borel measurable and bounded vector field on $\mathbb{R}^{d}$, such that $\nabla \cdot b$ (in distribution sense) is bounded and Borel measurable. Let $\Gamma_{F, L_{b}^{\star}}$ be the fundamental solution of the forward parabolic equation $\left(\frac{\partial}{\partial t}-L_{b}^{\star}\right) u=0$ (see [14, Definition, page 3]) and $\Gamma_{B, L_{b}}$ the fundamental solution of the backward parabolic equation $\left(\frac{\partial}{\partial t}+L_{b}\right) u=0$ (see [14, Definition, page 27]).

1) The following holds:

$$
p_{b}(\tau, \xi, t, x)=\Gamma_{B, L_{b}}(\xi, \tau ; x, t)=\Gamma_{F, L_{-b}-\nabla \cdot b}(x, t, \xi, \tau)
$$

for all $0 \leq \tau<t$ and $\xi, x \in \mathbb{R}^{d}$.

2) For given $\tau \geq 0, \varphi$ and $g$, the function

$$
w(x, t)=\int p_{b}(\tau, \xi, t, x) \varphi(\xi) d \xi+\int_{\tau}^{t} \int p_{b}(s, \xi, t, x) g(\xi, s) d \xi d s
$$

solves the initial value problem of the parabolic equation:

$$
\left(\frac{\partial}{\partial t}-L_{-b}-\nabla \cdot b\right) w=g, \quad w(\cdot, \tau)=\varphi .
$$


3) If in addition $b(x, t)$ is solenoidal, i.e. $\nabla \cdot b=0$, then

$$
p_{b}(\tau, \xi, t, x)=\Gamma_{F, L_{-b}}(x, t, \xi, \tau)
$$

for all $0 \leq \tau<t$ and $\xi, x \in \mathbb{R}^{d}$.

This lemma summarizes the representation formula of the solutions in terms of the fundamental solution and the relation between the fundamental solution and the transition probability density function for the associated diffusion process, which may be found in $[14,24]$ and [32] for example. The results in the previous lemma hold in fact under much weaker conditions on $b$ and can be generalised to a large class of elliptic operators, see $[1,3,14,24,32]$ and other standard literature on parabolic equations for details.

\subsection{An archetypical example}

Suppose the vorticity $\omega(x, t)$ of an incompressible fluid flow with velocity $u(x, t)$, without applying external force, always lies in the kernel of the rate-of-strain tensor, so that $\omega \cdot \nabla u=0$ identically, then the vorticity equation becomes

$$
\left(\frac{\partial}{\partial t}-L_{-u(x, t)}\right) \omega(x, t)=0
$$

with the initial data $\omega(\cdot, 0)=\omega_{0}$. Then, according to Lemma 1 ,

$$
\omega(x, t)=\int_{\mathbb{R}^{3}} p_{u}(0, y, t, x) \omega_{0}(y) d y .
$$

On the other hand, since $\Delta u=-\nabla \times \omega$, according to the Biot-Savart law,

$$
u(x, t)=\int_{\mathbb{R}^{3}} G(x-z) \times \omega(z, t) d z,
$$

where $G(x)=-\frac{1}{4 \pi} \frac{x}{|x|^{3}}$ is the vector valued singular kernel in $\mathbb{R}^{3}$. Since $\omega$ is a solution to (2.14), we are therefore able to rewrite the velocity field (2.16) in terms of the fundamental solution $p_{u}$, to obtain that

$$
\begin{aligned}
u(x, t) & =\int_{\mathbb{R}^{3}} \int_{\mathbb{R}^{3}} G(x-z) \times \omega_{0}(y) p_{u}(0, y, t, z) d z d y \\
& =\int_{\mathbb{R}^{3}}\left(\int_{\mathbb{R}^{3}} G(x-z) p_{u}(0, y, t, z) d z\right) \wedge \omega_{0}(y) d y \\
& =\int_{\mathbb{R}^{3}} \mathbb{E}[G(x-X(y, t))] \times \omega_{0}(y) d y,
\end{aligned}
$$

where $X(y, t)$ is the Taylor diffusion process with infinitesimal generator $L_{u}$ started at $y$ at $t=0$. That is, the solution to the stochastic differential equation

$$
d X(y, t)=u(X(y, t), t) d t+\sqrt{2 v} d B_{t}, \quad X(y, 0)=y,
$$

where $\left(B_{t}\right)_{t \geq 0}$ is a standard Brownian motion on a probability space $(\Omega, \mathscr{F}, \mathbb{P})$. Substituting (2.17) into (2.18), we may rewrite the previous stochastic differential equation as

$d X(x, t)=\left.\left(\int_{\mathbb{R}^{3}} \mathbb{E}[G(z-X(y, t))] \times \omega_{0}(y) d y\right)\right|_{z=X(x, t)} d t+\sqrt{2 v} d B_{t}, \quad X(x, 0)=x$, 
where $x$ runs through the state space $\mathbb{R}^{d}$. This is the archetypical example of the SDEs we are going to study in the present paper.

\subsection{Formulation of the problem}

Although our main examples come from the study of fluid dynamics, it will be beneficial formulating the problem in a more general setting. Still, we restrict our study to vector fields on Euclidean space $\mathbb{R}^{d}$. Though the methods and the results can be generalised to tensor fields with certain modifications.

Let $K(x)=\left(K_{j}^{i}(x)\right)$ be a $d \times d$ matrix-valued 'singular integral kernel, where $K_{j}^{i}$ are Borel measurable and locally integrable. We are interested in the following stochastic differential equation

$$
d X^{i}(x, t)=\left.\left(\int_{\mathbb{R}^{d}} \mathbb{E}\left[K_{j}^{i}(z-X(y, t))\right] \omega_{0}^{j}(y) d y\right)\right|_{z=X(x, t)} d t+d B_{t}^{i}, \quad X(x, 0)=x,
$$

where $i=1, \ldots, d, \omega_{0}=\left(\omega_{0}^{i}\right)$ is the initial data, and $B=\left(B^{i}\right)$ is a $d$-dimensional standard Brownian motion on some probability space. Before we carry out a study of this class of SDEs, let us reformulate (2.20) in a different form to facilitate our approach.

If $\mu$ is a measure on $\left(\mathbb{R}^{d}, \mathcal{B}\left(\mathbb{R}^{d}\right)\right)$, then $K \star \mu=\left(K_{j}^{i} \star \mu\right)$ denotes the convolution of $K$ and the measure $\mu$ where

$$
K_{j}^{i} \star \mu(x)=\int_{\mathbb{R}^{d}} K_{j}^{i}(x-y) \mu(d y)
$$

for $i, j=1, \ldots, d$, as long as the right-hand side is well defined.

If $U$ is an $\mathbb{R}^{d}$-valued random variable on some probability space $(\Omega, \mathscr{F}, \mathbb{P})$, then its distribution is denoted by $\mathscr{L}(U)$. By definition

$$
K_{j}^{i} \star \mathscr{L}(U)(x)=\mathbb{E}\left[K_{j}^{i}(x-U)\right] .
$$

If, in addition, the law of $U$ has a pdf $p(x)$, then

$$
K_{j}^{i} \star \mathscr{L}(U)(x)=\int_{\mathbb{R}^{d}} K_{j}^{i}(x-y) p(y) d y
$$

where the right-hand side is the convolution of $K$ and the function $p$.

After having introduced the basic data $K$ and $\omega_{0}$ and the notations, we are now in a position to reformulate the SDE we are going to study:

$$
d X^{i}(x, t)=\left[\int_{\mathbb{R}^{d}}\left(K_{j}^{i} \star \mathscr{L}(X(y, t))\right)(X(x, t)) \omega_{0}^{j}(y) d y\right] d t+d B_{t}^{i}
$$

with initial value $X(x, 0)=x$ for $x \in \mathbb{R}^{d}$ and $i=1, \ldots, d$.

The concepts of strong and weak solutions to (2.24) may be defined accordingly.

It will be convenient to introduce the following notations. If $Z=(Z(x, t))_{t \geq 0}$ is a family of continuous processes on some probability space, which is jointly continuous in $(x, t)$, then we may define a vector field denoted by $b_{Z}$ whose components are given by

$$
b_{Z}^{i}(x, t)=\int_{\mathbb{R}^{d}}\left(K_{j}^{i} \star \mathscr{L}(Z(y, t))\right)(x) \omega_{0}^{j}(y) d y,
$$

$i=1, \ldots, d$. Notice that by definition, $b_{Z}$ depends only on the one-dimensional marginal distributions of the process $(Z(y, t))_{t \geq 0}$. 
Suppose $b(x, t)$ is a time dependent vector field on $\mathbb{R}^{d}$, we may define another $t$-dependent vector field on $\mathbb{R}^{d}$, denoted by $K \diamond b(x, t)$, such that its $i$-th component is given by

$$
\int_{\mathbb{R}^{d}}\left(K_{j}^{i} \star \mathscr{L}(Z(y, t))\right)(x) \omega_{0}^{j}(y) d y,
$$

where $Z=(Z(y, t))_{t \geq 0}$ is the $L_{b}$-diffusion started at $y$ at the moment $t=0$, so that $K \diamond b=b_{Z}$. Since $Z(y, t)$ has a transition probability density $p_{b}(0, y, t, z)$, we can write

$$
(K \diamond b)^{i}(x, t)=\int_{\mathbb{R}^{d}}\left[\int_{\mathbb{R}^{d}} K_{j}^{i}(x-z) \omega_{0}^{j}(y) p_{b}(0, y, t, z) d z\right] d y .
$$

We therefore define the mapping $K \diamond$ which sends a vector field $b(x, t)$ to the vector field $K \diamond b(x, t)$. The non-linear mapping $b \rightarrow K \diamond b$ will play a crucial rôle in our study.

Under the above notations, we may rewrite $\operatorname{SDE}(2.24)$ as

$$
d X(x, t)=b_{X}(X(x, t), t) d t+d B_{t}, \quad X(x, 0)=x,
$$

for $x \in \mathbb{R}^{d}$. The following simple observation indeed leads to the approach we are going to develop in what follows.

Lemma 2 Let $b(x, t)$ be a bounded and Borel measurable vector field on $\mathbb{R}^{d}$, depending on time $t$. Suppose $K \diamond b=b$ and $(X, B)$ is a weak solution to the SDE

$$
d X^{i}(x, t)=b^{i}(X(x, t), t) d t+d B_{t}^{i}, \quad X(x, 0)=x,
$$

where $B$ is a standard Brownian motion on a probability space and $i=1, \ldots, d$. Then

$$
d X^{i}(x, t)=\left[\int_{\mathbb{R}^{d}}\left(K_{j}^{i} \star \mathscr{L}(X(y, t))\right)(X(x, t)) \omega_{0}^{j}(y) d y\right] d t+d B_{t}^{i}
$$

where $i=1, \ldots, d$. That is, $(X, B)$ is a weak solution to (2.24).

This lemma follows by definition: since $K \diamond b=b$, so $b=b_{X}$, which implies that

$$
\int_{\mathbb{R}^{d}}\left(K_{j}^{i} \star \mathscr{L}(X(y, t))\right)(x) \omega_{0}^{j}(y) d y=b^{i}(x, t)
$$

and therefore (2.30) follows from (2.29) immediately.

Example 3 If $d=3$ and $K=\left(K^{1}, K^{2}, K^{3}\right)$, then we set $K_{j}^{i}=\varepsilon^{i k j} K^{k}$ and $\operatorname{SDE}$ (2.30) becomes

$$
d X(x, t)=\left.\left(\int_{\mathbb{R}^{d}} \mathbb{E}[K(z-X(y, t))] \times \omega_{0}(y) d y\right)\right|_{z=X(x, t)} d t+d B_{t}, \quad X(x, 0)=x,
$$

which is the random vortex dynamical model, where $\omega_{0}$ represents the initial vorticity.

\section{Several facts about diffusions with bounded drifts}

In this section, we collect a few facts on diffusion processes and prove several technical estimates which will be used later in next section. Let $b(x, t)$ be a Borel measurable vector field on the Euclidean space $\mathbb{R}^{d}$, dependent on the time parameter $t \geq 0$. It is assumed that $b(x, t)$ is bounded: $|b(x, t)| \leq A$ for every $x$ and $t$, where $A$ is a non-negative constant. Then the unique $L_{b}$-diffusion (in the sense of weak solutions) may be constructed by using CameronMartin formula (see [33, Theorem 6.4.2, page 154]). Let $B=\left(B_{t}\right)_{t \geq 0}$ be a $d$-dimensional 
standard Brownian motion on $(\Omega, \mathcal{F}, \mathbb{P})$. Let $\mathcal{F}_{t}=\sigma\left\{B_{s}: s \leq t\right\}$ and $\mathcal{F}_{\infty}=\sigma\left\{B_{s}: s \geq 0\right\}$ be the natural filtration generated by this Brownian motion. Given $x$ and $\tau \geq 0$, define the exponential martingale called the Cameron-Martin density

$$
R_{b}(\tau, x, t)=e^{N_{b}(\tau, x, t)}
$$

where, for simplicity, we have written

$$
N_{b}(\tau, x, t):=\int_{\tau}^{t} b\left(r, B_{r}-B_{\tau}+x\right) d B_{r}-\frac{1}{2} \int_{\tau}^{t}|b|^{2}\left(r, B_{r}-B_{\tau}+x\right) d r
$$

for $t \geq \tau$. If $\tau=0$, then the symbol $\tau$ will be suppressed from the notations. Next, construct the probability $\mathbb{P}^{\tau, x}$ on $\left(\Omega, \mathcal{F}_{\infty}\right)$ such that

$$
\left.\frac{d \mathbb{P}^{\tau, x}}{d \mathbb{P}}\right|_{\mathcal{F}_{t}}=R_{b}(\tau, x, t)
$$

for all $t \geq \tau$. Then the family $\left\{\mathbb{P}^{\tau, x}: \tau \geq 0, x \in \mathbb{R}^{d}\right\}$ on $\left(\Omega, \mathcal{F}_{\infty}\right)$ is a diffusion family with generator $L_{b}$ (see for example [17,33]). In particular

$$
\int_{\mathbb{R}^{d}} f(y) p_{b}(\tau, x, t, y) d y=\mathbb{P}\left[R_{b}(\tau, x, t) f\left(B_{t}-B_{\tau}\right)\right]
$$

for any Borel function $f$, as long as one of the integrals in the equation makes sense, where $p_{b}(\tau, x, t, y)$ is the transition probability density function of the $L_{b}$-diffusion. It is known (see for example [32]) that $p_{b}(\tau, x, t, y)$ is positive and continuous on any $t>\tau \geq 0$ and $x, y \in \mathbb{R}^{d}$. Moreover, for every $T>0$, there is a constant $M$ depending on $A, d$ and $T$ only, such that

$$
\frac{1}{M t^{d / 2}} e^{-M \frac{|y-x|^{2}}{t}} \leq p_{b}(\tau, x, \tau+t, y) \leq \frac{M}{t^{d / 2}} e^{-\frac{|y-x|^{2}}{M t}}
$$

for all $\tau \geq 0$ and $T \geq t>0$. This is the so-called Aronson estimate (see [2,31,32] for example). In our study, we need more precise information about the constant $M$, which was obtained in [27,28].

Lemma 4 If the vector field $b(x, t)$ is bounded and Borel measurable, and $p_{b}$ is defined as above, then there is a positive universal constant $\kappa$, depending only on the dimension $d$ and $1<q<\frac{d}{d-1}$, such that

$$
p_{b}(\tau, x, \tau+t, y) \leq \frac{1}{(2 \pi t)^{d / 2}} e^{-\frac{|x-y|^{2}}{2 t}}\left(1+\kappa A(\sqrt{t}+|x-y|) e^{\frac{q-1}{2 q t}|x-y|^{2}+\frac{A^{2} t}{2(q-1)}}\right)
$$

for all $x, y \in \mathbb{R}^{d}, \tau \geq 0$ and $t>0$.

As a consequence, we establish the following estimate, which will play a crucial rôle in the proof of our main theorem.

Lemma 5 Let $f \in L^{\infty}\left(\mathbb{R}^{d}\right), \gamma \in[0, d)$ and $\rho>0$. Define

$$
I(f, x, t, \rho, \gamma)=\int_{\mathbb{R}^{d}} \int_{\{|z|<\rho\}} \frac{1}{|z|^{\gamma}}|f(y)| p_{b}(0, y, t, x-z) d z d y
$$

for any $x \in \mathbb{R}^{d}$ and $t>0$. Then there exists a universal positive constant $\kappa_{1}$ depending only ond, such that

$$
I(f, x, t, \rho, \gamma) \leq \frac{\rho^{d-\gamma}}{d-\gamma} \kappa_{1}\|f\|_{\infty}\left(1+A \sqrt{t} e^{\frac{A^{2} t}{2(q-1)}}\right)
$$

for all $x$ and $t>0$. 
Proof Without losing generality, we may assume that $f \geq 0$. Using the sharp estimate (3.6), we have

$$
\begin{aligned}
& I(f, x, t, \rho, \gamma) \leq \int_{\mathbb{R}^{d}} \int_{\{|z|<\rho\}} \frac{f(y)}{|z|^{\gamma}} \frac{e^{-\frac{|y-x+z|^{2}}{2 t}}}{(2 \pi t)^{d / 2}} \\
& \times\left(1+\kappa A(\sqrt{t}+|y-x+z|) e^{\frac{q-1}{2 q t}|y-x+z|^{2}+\frac{A^{2} t}{2(q-1)}}\right) d z d y \\
& =\int_{\mathbb{R}^{d}} \int_{\{|z|<\rho\}} \frac{f(y+x-z)}{|z|^{\gamma}} \frac{e^{-\frac{|y|^{2}}{2 t}}}{(2 \pi t)^{d / 2}}\left(1+\kappa A(\sqrt{t}+|y|) e^{\frac{q-1}{2 q t}|y|^{2}+\frac{A^{2} t}{2(q-1)}}\right) d z d y \\
& \leq\|f\|_{\infty}\left(\int_{\{|z|<\rho\}} \frac{1}{|z|^{\gamma}} d z\right)\left(1+\kappa A \sqrt{t} e^{\frac{A^{2} t}{2(q-1)}} \int_{\mathbb{R}^{d}} \frac{e^{-\frac{|y|^{2}}{2 t}}}{(2 \pi t)^{d / 2}}\left(1+\frac{|y|}{\sqrt{t}}\right) e^{\frac{q-1}{2 q t}|y|^{2}} d y\right) \\
& =\|f\|_{\infty}\left(\int_{\{|z|<\rho\}} \frac{1}{|z|^{\gamma}} d z\right)\left(1+\kappa A \sqrt{t} e^{\frac{A^{2} t}{2(q-1)}} \int_{\mathbb{R}^{d}} \frac{e^{-\frac{|y|^{2}}{2}}}{(2 \pi)^{d / 2}}(1+|y|) e^{\frac{q-1}{2 q}|y|^{2}} d y\right) \\
& \leq \frac{\rho^{d-\gamma}}{d-\gamma} \kappa_{1}\|f\|_{\infty}\left(1+A \sqrt{t} e^{\frac{A^{2} t}{2(q-1)}}\right)
\end{aligned}
$$

where

$$
\kappa_{1}=\max \left\{\operatorname{Vol}\left(S^{d-1}\right), \kappa \int_{\mathbb{R}^{d}}(1+|y|) \frac{e^{-\frac{|y|^{2}}{2 q}}}{(2 \pi)^{d / 2}} d y\right\}
$$

and the proof is complete.

We also need the following estimate which is completely elementary though.

Lemma 6 Let $f \in L^{1}\left(\mathbb{R}^{d}\right)$ and $\rho>0, \gamma \geq 0$ be two constants. Define

$$
J(f, x, t, \rho, \gamma)=\int_{\mathbb{R}^{d}} \int_{\{|z| \geq \rho\}} \frac{1}{|z|^{\gamma}}|f(y)| p_{b}(0, y, t, x-z) d z d y
$$

for all $x \in \mathbb{R}^{d}$ and $t>0$. Then

$$
J(f, x, t, \rho, \gamma) \leq \frac{1}{\rho^{\gamma}}\|f\|_{L^{1}}
$$

for all $x$ and $t>0, \rho>0$ and $\gamma \geq 0$.

Proof Since $\gamma \geq 0$, so that

$$
\begin{aligned}
J(f, x, t, \rho, \gamma) & \leq \frac{1}{\rho^{\gamma}} \int_{\mathbb{R}^{d}} \int_{\{|z| \geq \rho\}}|f(y)| p_{b}(0, y, t, x-z) d z d y \\
& \leq \frac{1}{\rho^{\gamma}} \int_{\mathbb{R}^{d}} \int_{\mathbb{R}^{d}}|f(y)| p_{b}(0, y, t, x-z) d z d y \\
& =\frac{1}{\rho^{\gamma}} \int_{\mathbb{R}^{d}} \int_{\mathbb{R}^{d}}|f(y)| p_{b}(0, y, t, z) d z d y \\
& =\frac{1}{\rho^{\gamma}} \int_{\mathbb{R}^{d}}|f(y)| d y \\
& =\frac{1}{\rho^{\gamma}}\|f\|_{L^{1}}
\end{aligned}
$$

and the proof is complete. 


\section{Weak solutions}

In this section we prove under certain conditions that there is a unique weak solution to (2.24).

To this end we make several assumptions on $\omega_{0}$ and $K$, Hypotheses 1 and Hypotheses 2, which will be in force throughout the remainder of the paper.

Hypothesise 1 . There are constants $C_{0} \geq, \gamma_{1} \in[0, d)$ and $\gamma_{2} \geq 0$, such that $K=\left(K_{j}^{i}\right)$ satisfies the following growth condition: there are two constants $\gamma_{1}$ and $\gamma_{2} \geq 0$ such that

$$
|K(x)| \leq \frac{C_{0}}{|x|^{\gamma_{1}}} \quad \text { for all } x \neq 0 \text { and }|x|<1,
$$

and

$$
|K(x)| \leq \frac{C_{0}}{|x|^{\gamma_{2}}} \quad \text { for all }|x| \geq 1
$$

Hypothesise 2. There are two non-negative constants $C_{1}$ and $C_{\infty}$, such that the initial vorticity $\omega_{0}$ is bounded and integrable with $\left\|\omega_{0}\right\|_{L^{1}} \leq C_{1}$ and $\left\|\omega_{0}\right\|_{\infty} \leq C_{\infty}$.

Choose and fix a number $q \in\left(1, \frac{d}{d-1}\right)$ and set

$$
C_{K}=C_{0}\left(\frac{\kappa_{1} C_{\infty}}{d-\gamma_{1}}\left(1+e^{\frac{1}{2(q-1)}}\right)+C_{1}\right) \text { and } T_{K}=\frac{1}{C_{K}^{2}} .
$$

The crucial fact about $C_{K}$ and $T_{K}$ is that they depend on $C_{0}, C_{1}, C_{\infty}$ and $\gamma_{1}$ only.

Lemma 7 If $b(x, t)$ is a time-dependent vector field such that $|b(x, t)| \leq C_{K}$ for all $x \in \mathbb{R}^{d}$ and $t \leq T_{K}$, then $K \diamond b$ is also bounded with the same bound. That is $|K \diamond b(x, t)| \leq C_{K}$ for all $x \in \mathbb{R}^{d}$ and $t \leq T_{K}$.

Proof Let $B=\left\{z \in \mathbb{R}^{d}:|z|<1\right\}$. Then

$$
\begin{aligned}
|K \diamond b(x, t)|= & \mid \int_{\mathbb{R}^{d}} \int_{B} K(z) \omega_{0}(y) p_{b}(0, y, t, x-z) d z d y \\
& +\int_{\mathbb{R}^{d}} \int_{\mathbb{R}^{d} \backslash B} K(z) \omega_{0}(y) p_{b}(0, y, t, x-z) d z d y \mid \\
\leq & \int_{\mathbb{R}^{d}} \int_{B}|K(z)|\left|\omega_{0}(y)\right| p_{b}(0, y, t, x-z) d z d y \\
& +\int_{\mathbb{R}^{d}}\left|\omega_{0}(y)\right| \int_{\mathbb{R}^{d} \backslash B}|K(z)| p_{b}(0, y, t, x-z) d z d y \\
= & : I_{1}+I_{2}
\end{aligned}
$$

The estimate for $I_{1}$ follows directly from Lemma 5 :

$$
\begin{aligned}
I_{1} & \leq C_{0} I\left(\omega_{0}, x, t, 1, \gamma_{1}\right) \\
& \leq \frac{\kappa_{1} C_{0} C_{\infty}}{d-\gamma_{1}}\left(1+A \sqrt{t} e^{\frac{A^{2} t}{2(q-1)}}\right)
\end{aligned}
$$

where $A=C_{K}$. By Lemma 6 we deduce that

$$
I_{2} \leq C_{0} J\left(\omega_{0}, x, t, 1, \gamma_{2}\right) \leq C_{0} C_{1} .
$$

Putting the estimates for $I_{1}$ and $I_{2}$ together we may conclude that

$$
|K \diamond b(x, t)| \leq \frac{\kappa_{1} C_{0} C_{\infty}}{d-\gamma_{1}}\left(1+A \sqrt{t} e^{\frac{A^{2} t}{2(q-1)}}\right)+C_{0} C_{1}
$$


for all $x \in \mathbb{R}^{d}$ and $t \geq 0$. Since $A \sqrt{t} \leq 1$ for any $t \leq T_{K}$, we therefore have

$$
|K \diamond b(x, t)| \leq \frac{\kappa_{1} C_{0} C_{\infty}}{d-\gamma_{1}}\left(1+e^{\frac{1}{2(q-1)}}\right)+C_{0} C_{1}
$$

for any $x$ and $t \leq T_{K}$. The conclusion then follows immediately from the definition of $C_{K}$ and $T_{K}$.

Next we are going to establish another key estimate for the mapping $b \rightarrow K \diamond b$, where $b(x, t)$ are vector fields such that $|b(x, t)| \leq C_{K}$ for any $t \leq T_{K}$.

Lemma 8 There exists a positive constant $C_{L}$ depending only on $C_{0}, C_{1}, C_{\infty}$, such that for any $b(x, t)$ and $\tilde{b}(x, t)$ satisfying that $|b(x, t)| \leq C_{K}$ and $|\tilde{b}(x, t)| \leq C_{K}$ for all $x$ and $t \leq T_{K}$ we have

$$
|K \diamond b(x, t)-K \diamond \tilde{b}(x, t)| \leq(t+\sqrt{t}) C_{L}\|b-\tilde{b}\|_{L^{\infty}\left(\mathbb{R}^{d},[0, t]\right)}
$$

for all $x$ and $t \leq T_{K}$.

Proof We prove this by using Cameron-Martin formula [33, Theorem 6.4.2, page 154]. Let $B$ be a $d$-dimensional standard Brownian motion on some probability space $(\Omega, \mathcal{F}, \mathbb{P})$ and $R_{c}(x, t)=e^{N_{c}(x, t)}$ be the Cameron-Martin density (see (3.1) and (3.2)) with respect to the vector field $c$ starting at $x$ at the moment 0 . Then

$$
\begin{aligned}
N_{b}(x, t)-N_{\tilde{b}}(x, t)= & \int_{0}^{t}(b-\tilde{b})\left(r, B_{r}+x\right) d B_{r} \\
& -\frac{1}{2} \int_{0}^{t}\left(|b|^{2}-|\tilde{b}|^{2}\right)\left(r, B_{r}+x\right) d r \\
& =M(x, t)+A(x, t),
\end{aligned}
$$

where

$$
M(x, t):=\int_{0}^{t}(b-\tilde{b})\left(r, B_{r}+x\right) d B_{r},
$$

whose quadratic process

$$
\langle M\rangle_{t}=\int_{0}^{t}|b-\tilde{b}|^{2}\left(r, B_{r}+x\right) d r \leq\|b-\tilde{b}\|_{\infty}^{2} t,
$$

and

$$
\begin{aligned}
A(x, t) & :=-\frac{1}{2} \int_{0}^{t}\left(|b|^{2}-|\tilde{b}|^{2}\right)\left(r, B_{r}+x\right) d r \\
& =-\frac{1}{2} \int_{0}^{t}(b-\tilde{b})(b+\tilde{b})\left(r, B_{r}+x\right) d r .
\end{aligned}
$$

It is clear that

$$
|A(x, t)| \leq C_{K} t\|b-\tilde{b}\|_{L^{\infty}([0, t])}
$$

and therefore,

$$
\left|N_{b}(x, t)-N_{\tilde{b}}(x, t)\right| \leq\left|M_{t}\right|+C_{K} t\|b-\tilde{b}\|_{L^{\infty}([0, t])} .
$$


Now we write

$$
\begin{aligned}
R_{b}-R_{\tilde{b}} & =\int_{0}^{1} \frac{d}{d s} e^{(1-s) N_{\tilde{b}}+s N_{b}} d s \\
& =\int_{0}^{1} e^{(1-s) N_{\tilde{b}}+s N_{b}}\left(N_{b}-N_{\tilde{b}}\right) d s .
\end{aligned}
$$

Let $b_{s}=(1-s) \tilde{b}+s b$ for $s \in[0,1]$. Then $\left|b_{s}(x, t)\right| \leq C_{K}$ and

$$
(1-s) N_{\tilde{b}}+s N_{b}=N_{b_{s}}-(1-s) s|b-\tilde{b}|^{2} .
$$

Substituting this equality into (4.9) to obtain

$$
R_{b}-R_{\tilde{b}}=\int_{0}^{1} R_{b_{s}} e^{-(1-s) s|b-\tilde{b}|^{2}}\left(N_{b}-N_{\tilde{b}}\right) d s .
$$

Now we are in a position to study the non-linear mapping $c \rightarrow K \diamond c$. According to CameronMartin formula

$$
\begin{aligned}
K \diamond c(x, t) & =\int_{\mathbb{R}^{d}}\left[\int_{\mathbb{R}^{d}} K(x-z) \omega_{0}(y) p_{c}(0, y, t, z) d z\right] d y \\
& =\int_{\mathbb{R}^{d}} \mathbb{E}\left[R_{c}(y, t) K\left(x-B_{t}-y\right)\right] \omega_{0}(y) d y .
\end{aligned}
$$

Split $K$ into a sum $K=K_{1}+K_{2}$ where

$$
K_{1}(z)=1_{\{|z|<1\}} K(z) \text { and } K_{2}(z)=1_{\{|z| \geq 1\}} K(z) .
$$

Then

$$
\begin{aligned}
K \diamond c(x, t)= & \int_{\mathbb{R}^{d}} \mathbb{E}\left[R_{c}(y, t) K_{1}\left(x-B_{t}-y\right)\right] \omega_{0}(y) d y \\
& +\int_{\mathbb{R}^{d}} \mathbb{E}\left[R_{c}(y, t) K_{2}\left(x-B_{t}-y\right)\right] \omega_{0}(y) d y .
\end{aligned}
$$

Let

$$
D(x, t):=K \diamond b(x, t)-K \diamond \tilde{b}(x, t) .
$$

Then by using the previous formula for $K \diamond c$ we have

$$
\begin{aligned}
D(x, t)= & \int_{\mathbb{R}^{d}} \mathbb{E}\left[\left(R_{b}(y, t)-R_{\tilde{b}}(y, t)\right) K_{1}\left(x-B_{t}-y\right)\right] \omega_{0}(y) d y \\
& +\int_{\mathbb{R}^{d}} \mathbb{E}\left[\left(R_{b}(y, t)-R_{\tilde{b}}(y, t)\right) K_{2}\left(x-B_{t}-y\right)\right] \omega_{0}(y) d y \\
= & : J_{1}+J_{2} .
\end{aligned}
$$

Substituting (4.10) into $J_{1}$, we may write

$J_{1}=\int_{\mathbb{R}^{d}} \mathbb{E}\left[\left(\int_{0}^{1} R_{b_{s}}(y, t) e^{-(1-s) s|b-\tilde{b}|^{2}}\left(N_{b}(y, t)-N_{\tilde{b}}(y, t)\right) d s\right) K_{1}\left(x-B_{t}-y\right)\right] \omega_{0}(y) d y$ and therefore 


$$
\begin{aligned}
\left|J_{1}\right| \leq & \int_{0}^{1} \int_{\mathbb{R}^{d}} \mathbb{E}\left[R_{b_{s}}(y, t)\left|N_{b}(y, t)-N_{\tilde{b}}(y, t)\right|\left|K_{1}\left(x-B_{t}-y\right)\right|\right]\left|\omega_{0}(y)\right| d y d s \\
\leq & C_{0} \int_{0}^{1} \int_{\mathbb{R}^{d}} \mathbb{E}\left[R_{b_{s}}(y, t) \frac{1_{\left\{\left|x-B_{t}-y\right|<1\right\}}}{\left|x-B_{t}-y\right|^{\gamma_{1}}}\left|M_{t}\right|\right]\left|\omega_{0}(y)\right| d y d s \\
& +C_{0} C_{K} t\|b-\tilde{b}\|_{L^{\infty}([0, t])} \int_{0}^{1} \int_{\mathbb{R}^{d}} \mathbb{E}\left[R_{b_{s}}(y, t) \frac{1_{\left\{\left|x-B_{t}-y\right|<1\right\}}}{\left|x-B_{t}-y\right|^{\gamma_{1}}}\right]\left|\omega_{0}(y)\right| d y d s \\
= & : J_{1,1}+J_{1,2},
\end{aligned}
$$

where the second inequality comes from (4.8).

To deal with $J_{1,1}$, choose and fix $\alpha, \beta>1$ such that $\alpha \gamma_{1}<d$ and $\alpha^{-1}+\beta^{-1}=1$. Then

$$
R_{b_{s}}^{\alpha}=R_{\alpha b_{s}} e^{\frac{\alpha^{2}-1}{2} \int_{0}^{t}\left|b_{s}\right|^{2} d r} \leq e^{\frac{\alpha^{2}-1}{2} C_{K}^{2} t} R_{\alpha b_{s}} .
$$

Also, by using Burkholder-Davis-Gundy inequality (see for example [16, Theorem 3.1, page 110]),

$$
\mathbb{E}\left[\left|M_{t}\right|^{\beta}\right] \leq C_{\beta} \mathbb{E}\left[\langle M\rangle_{t}^{\frac{\beta}{2}}\right] \leq C_{\beta} t^{\frac{\beta}{2}}\|b-\tilde{b}\|_{L^{\infty}\left(\mathbb{R}^{d} \times[0, t]\right)}^{\beta} .
$$

Thus, by applying Hölder's inequality in $J_{1,1}$, we deduce that

$$
\begin{aligned}
& J_{1,1}=C_{0} \int_{0}^{1} \int_{\mathbb{R}^{d}} \mathbb{E}\left[R_{b_{s}}(y, t) \frac{1_{\left\{\left|x-B_{t}-y\right|<1\right\}}}{\left|x-B_{t}-y\right|^{\gamma_{1}}}\left|M_{t}\right|\right]\left|\omega_{0}(y)\right| d y d s \\
& \leq C_{0} \int_{0}^{1} \sqrt[\alpha]{\int_{\mathbb{R}^{d}} \mathbb{E}\left[R_{b_{s}}^{\alpha}(y, t) \frac{1_{\left\{\left|x-B_{t}-y\right|<1\right\}}}{\left|x-B_{t}-y\right|^{\alpha \gamma_{1}}}\right]\left|\omega_{0}(y)\right| d y} \\
& \times \sqrt[\beta]{\int_{\mathbb{R}^{d}} \mathbb{E}\left[\left|M_{t}\right|^{\beta}\right]\left|\omega_{0}(y)\right| d y d s}
\end{aligned}
$$

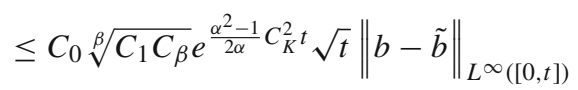

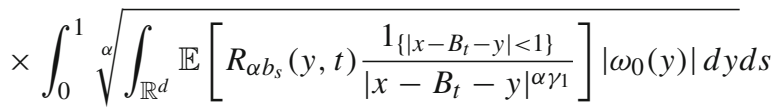

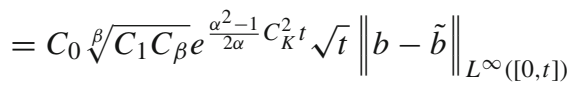

$$
\begin{aligned}
& \times \int_{0}^{1} \sqrt[\alpha]{\int_{\mathbb{R}^{d}} \int_{|z|<1} \frac{1}{|z|^{\alpha \gamma_{1}}} p_{\alpha b_{s}}(0, y, t, x-z) d z\left|\omega_{0}(y)\right| d y} d s \\
& \leq C_{0} \sqrt[\beta]{C_{1} C_{\beta}} e^{\frac{\alpha^{2}-1}{2 \alpha} C_{K}^{2} t} \sqrt[\alpha]{\frac{\kappa_{1}\left(1+\alpha C_{K} \sqrt{t} e^{\frac{\alpha^{2} C_{K}^{2}}{2(q-1)}}\right)}{d-\alpha \gamma_{1}}} \sqrt{t}\|b-\tilde{b}\|_{L^{\infty}\left(\mathbb{R}^{d} \times[0, t]\right)} \\
& \leq C_{0} \sqrt[\beta]{C_{1} C_{\beta}} e^{\frac{\alpha^{2}-1}{2 \alpha}} \sqrt[\alpha]{\frac{\kappa_{1}\left(1+\alpha e^{\left.\frac{\alpha^{2}}{2(q-1)}\right)}\right.}{d-\alpha \gamma_{1}}} \sqrt{t}\|b-\tilde{b}\|_{L^{\infty}\left(\mathbb{R}^{d} \times[0, t]\right)},
\end{aligned}
$$

for all $t \leq T_{K}$, where the second inequality follows from (4.11), the third inequality follows from Lemma 5, and the last inequality follows from the fact that $C_{K} t \leq 1$ for all $t \leq T_{K}$. 
To deal with $J_{1,2}$, we apply Lemma 6 and obtain that

$$
\begin{aligned}
J_{1,2}= & C_{0} C_{K} t\|b-\tilde{b}\|_{L^{\infty}([0, t])} \\
& \times \int_{0}^{1} \int_{\mathbb{R}^{d}} \mathbb{E}\left[R_{b_{s}}(y, t) \frac{1_{\left\{\left|x-B_{t}-y\right|<1\right\}}}{\left|x-B_{t}-y\right|^{\gamma_{1}}}\right]\left|\omega_{0}(y)\right| d y d s \\
= & C_{0} C_{K} t\|b-\tilde{b}\|_{L^{\infty}([0, t])} \\
& \times \int_{0}^{1} \int_{\mathbb{R}^{d}}\left(\int_{\{|z|<1\}} \frac{1}{|z|^{\gamma_{1}}} p_{b_{s}}(0, y, t, x-z) d z\right)\left|\omega_{0}(y)\right| d y d s \\
\leq & \frac{C_{0} C_{\infty} C_{K} \kappa_{1}}{d-\gamma_{1}}\left(1+C_{K} \sqrt{t} e^{\frac{C_{K}^{2}}{2(q-1)}}\right) t\|b-\tilde{b}\|_{L^{\infty}\left(\mathbb{R}^{d} \times[0, t]\right)} \\
\leq & \frac{C_{0} C_{\infty} C_{K} \kappa_{1}}{d-\gamma_{1}}\left(1+e^{\frac{1}{2(q-1)}}\right) t\|b-\tilde{b}\|_{L^{\infty}\left(\mathbb{R}^{d} \times[0, t]\right)}
\end{aligned}
$$

for any $t \leq T_{K}$, where the first inequality follows from the estimate in Lemma 6 .

Now we treat with $J_{2}$. Since

$$
J_{2}=\int_{\mathbb{R}^{d}} \mathbb{E}\left[\left(\int_{0}^{1} R_{b_{s}}(y, t) e^{-(1-s) s|b-\tilde{b}|^{2}}\left(N_{b}(y, t)-N_{\tilde{b}}(y, t)\right) d s\right) K_{2}\left(x-B_{t}-y\right)\right] \omega_{0}(y) d y
$$

and $\left|K_{2}(z)\right| \leq C_{0}$, so by (4.8) we have

$$
\begin{aligned}
& \left|J_{2}\right| \leq C_{0} \int_{0}^{1} \int_{\mathbb{R}^{d}} \mathbb{E}\left[R_{b_{s}}(y, t)\left|M_{t}\right|\right]\left|\omega_{0}(y)\right| d y d s \\
& +C_{0} C_{1} C_{K} t\|b-\tilde{b}\|_{L^{\infty}\left(\mathbb{R}^{d} \times[0, t]\right)} \\
& \leq C_{0} \int_{0}^{1} \int_{\mathbb{R}^{d}} \sqrt{\mathbb{E}\left[\left|M_{t}\right|^{2}\right]} \sqrt{\mathbb{E}\left[R_{b_{s}}^{2}(y, t)\right]}\left|\omega_{0}(y)\right| d y d s \\
& +C_{0} C_{1} C_{K} t\|b-\tilde{b}\|_{L^{\infty}\left(\mathbb{R}^{d} \times[0, t]\right)} \\
& \leq C_{0} C_{1} \sqrt{e^{\frac{\alpha^{2}-1}{2}}} \sqrt{t}\|b-\tilde{b}\|_{L^{\infty}([0, t])} \\
& +C_{0} C_{1} C_{K} t\|b-\tilde{b}\|_{L^{\infty}\left(\mathbb{R}^{d} \times[0, t]\right)},
\end{aligned}
$$

where the last inequality comes from (4.11). Putting these estimates for $J_{1}$ and $J_{2}$ together, we deduce (4.5) with a positive constant $C_{L}$ which depends only on the structure constants $C_{0}, C_{1}, C_{\infty}, \gamma_{1}$ and $d$ (as $\alpha$ and $q$ are constants depending only on $d$ and $\gamma_{1}$ ). For example

$$
\begin{aligned}
C_{L}= & C_{0} C_{1} \sqrt{e^{\frac{\alpha^{2}-1}{2}}}\left(C_{K}+1\right) \\
& +C_{0} C_{\infty} C_{K} \frac{1}{d-\gamma_{1}} \kappa_{1}\left(1+e^{\frac{1}{2(q-1)}}\right) \\
& +C_{0} \sqrt[\beta]{C_{1} C_{\beta}} e^{\frac{\alpha^{2}-1}{2 \alpha}} \sqrt[\alpha]{\frac{1}{d-\alpha \gamma_{1}} \kappa_{1}\left(1+\alpha e^{\frac{\alpha^{2}}{2(q-1)}}\right)}
\end{aligned}
$$

will do.

We are now in a position to prove the main result about weak solutions to (2.24). 
Theorem 9 There exist two positive constants $T_{L}$ and $C_{K}$ depending on $C_{0}, C_{1}, C_{\infty}, \gamma_{1} \in$ $[0, d)$ and $d$ only such that the followings hold:

1) The (non-linear) mapping $b \rightarrow K \diamond b$ is contractive on the space of bounded timedependent vector fields. More precisely

$$
\|K \diamond b-K \diamond \tilde{b}\|_{\infty} \leq \frac{1}{2}\|b-\tilde{b}\|_{\infty}
$$

for any vector fields $b$ and $\tilde{b}$ such that $\|b\|_{\infty} \leq C_{K}$, $\|\tilde{b}\|_{\infty} \leq C_{K}$, where $\|b\|_{\infty}=$ $\|b\|_{L^{\infty}\left(\mathbb{R}^{d} \times\left[0, T_{L}\right]\right)}$. Hence, there is a unique b such that $K \diamond b=b$.

2) There is a unique weak solution $(X, B)$ on some probability space to the SDE (2.24) up to time $T_{L}$, where $B$ is a Brownian motion and $X$ satisfies (2.24), and

$$
b^{i}(\cdot, t)=\int_{\mathbb{R}^{d}}\left(K_{j}^{i} \star \mathscr{L}(X(y, t))\right) \omega_{0}^{j}(y) d y
$$

is bounded for $i=1, \cdots, d$.

Proof Choose $T_{L}=\frac{1}{4} C_{L} \wedge 1$ where $C_{L}$ is given by (4.13). Then (4.14) follows immediately.

The second part then follows from Lemma 2.

We finish this section with a comment on the global solutions of (2.24). As long as $K$ is a singular integral kernel, bounded at infinity, we have shown that there is a unique weak solution to (2.24) with initial data $\omega_{0} \in L^{1}\left(\mathbb{R}^{d}\right) \cap L^{\infty}\left(\mathbb{R}^{d}\right)$ for the time duration $\left[0, T_{L}\right]$, where $T_{L}$ depends only on the structure constants $C_{i}(i=0,1, \infty)$ and $\gamma_{1} \in[0, d)$. However, we are unable to conclude that the weak solution exists for all time $t$. The reason is that the SDE (2.24) does not define a dynamical system, which is not proposed as an initial value problem.

Finally, we should point out that we do not claim, although we strongly believe it is not the case, if $\omega_{0}$ and $K$ are regular enough, there are other fixed vector fields $c$ in the sense that $K \diamond c=c$ but $c(x, t)$ is unbounded on some time interval $[0, T]$.

\section{Strong solutions}

With the same assumptions on $K$ and $\omega_{0}$ as in Sect. 4, we show that there is a weak solution to (2.24) by using the result in [39] for multi-dimensional diffusion processes with bounded drifts. Moreover, under a growth condition on $K$, we are able to show the Hölder continuity of the vector field $K \diamond b$.

Firstly, by using the results in Zvonkin-Krylov [41] and Veretennikov [39] we deduce the following.

Theorem 10 Let $B=\left(B_{t}\right)_{t \geq 0}$ be ad-dimensional standard Brownian motion on a probability space $(\Omega, \mathcal{F}, \mathbb{P})$. There is a unique family of stochastic processes $X(x, t)$ which is jointly continuous in $(x, t)$ almost surely, and satisfies the stochastic integral equations

$$
X^{i}(x, t)=x+\int_{0}^{t}\left[\int_{\mathbb{R}^{d}}\left(K_{j}^{i} \star \mathscr{L}(X(y, s))\right)(X(x, s)) \omega_{0}^{j}(y) d y\right] d s+B_{t}^{i}
$$

for $t \in\left[0, T_{L}\right]$, and

$$
b^{i}(\cdot, t)=\int_{\mathbb{R}^{d}}\left(K_{j}^{i} \star \mathscr{L}(X(y, s))\right) \omega_{0}^{j}(y) d y
$$

where $i=1, \ldots, d$, are bounded. 
Proof According to Theorem 9, for any $t \leq T_{L}$ (and extended it to be zero for $t>T_{L}$ ), there is a unique bounded vector field $b(x, t)$ satisfying $K \diamond b=b$. Since $b$ is bounded and Borel measurable, then there is a unique strong solution $X(x, t)$ to the ordinary stochastic differential equation

$$
d X(x, t)=b(X(x, t), t) d t+d B_{t}, \quad X(x, 0)=x
$$

(see Veretennikov [39, Theorem 1, page 388]). Hence, by Lemma 2, $X(x, t)$ is the unique strong solution to (2.24).

We are going to show that the vector field (5.1) is in fact Hölder continuous. To this end, we need the following Hölder continuity result of the transition probability density function, proved originally by Nash [23] and later by Aronson [2], Fabes and Stroock [13]. We take this from [31, Theorem II.2.12, page 340].

Lemma 11 Under the same assumption as in Lemma 4, there are constants $C_{H}>0$ and $\alpha \in(0,1)$ depending only on $A$ and $d$ such that

$$
\left|p_{b}(s, y, t, x)-p_{b}(s, y, \tilde{t}, \tilde{x})\right| \leq \frac{C_{H}}{\delta^{3}}\left(\frac{|t-\tilde{t}|^{\frac{1}{2}} \vee|x-\tilde{x}|}{\delta}\right)^{\alpha}
$$

for all $s \geq 0, \delta^{2} \leq t-s \leq \frac{1}{\delta^{2}},|x-\tilde{x}| \leq \delta$, for any $\delta>0$.

Lemma 12 Under the same assumptions for $K$ and $\omega_{0}$ as in the previous section, we further assume $\gamma_{1}=\gamma_{2} \equiv \gamma$, which belongs to $[0, d)$. Suppose $|b(x, t)| \leq C_{K}$ for all $x$ and $t$. Then $K \diamond b(x, t)$ is Hölder continuous on any compact subset of $\mathbb{R}^{d} \times\left(0, T_{K}\right]$, where the Hölder exponent and Hölder norm depend only on $C_{K}$.

Proof By using Lemma 11, if $T_{K} \geq t, \tilde{t}>\delta^{2}$ and $|x-\tilde{x}|<\delta$ (for $\delta>0$ small enough), for simplicity set

$$
H=|K \diamond b(x, t)-K \diamond b(\tilde{x}, \tilde{t})| .
$$

Then

$$
\begin{aligned}
H= & \left|\int_{\mathbb{R}^{d}}\left[\int_{\mathbb{R}^{d}} K(z) \omega_{0}(y)\left(p_{b}(0, y, t, x-z)-p_{b}(0, y, \tilde{t}, \tilde{x}-z)\right) d z\right] d y\right| \\
\leq & \int_{\mathbb{R}^{d}}\left[\int_{\mathbb{R}^{d}}|K(z)|\left|\omega_{0}(y)\right|\left|p_{b}(0, y, t, x-z)-p_{b}(0, y, \tilde{t}, \tilde{x}-z)\right| d z\right] d y \\
\leq & \int_{\mathbb{R}^{d}}\left[\int_{\{|z|<\rho\}}|K(z)|\left|\omega_{0}(y)\right|\left|p_{b}(0, y, t, x-z)-p_{b}(0, y, \tilde{t}, \tilde{x}-z)\right| d z\right] d y \\
& +\int_{\mathbb{R}^{d}}\left[\int_{\{|z| \geq \rho\}}|K(z)|\left|\omega_{0}(y)\right|\left|p_{b}(0, y, t, x-z)-p_{b}(0, y, \tilde{t}, \tilde{x}-z)\right| d z\right] d y \\
\leq & C_{1}\left\{\frac{C_{H}}{\delta^{d}}\left(\frac{|t-\tilde{t}|^{\frac{1}{2}} \vee|x-\tilde{x}|}{\delta}\right)^{\alpha}\right\} \frac{C_{0}}{d-\gamma} \rho^{d-\gamma}+2 \frac{C_{0} C_{1}}{\rho^{\gamma}}
\end{aligned}
$$

for any $\rho>0$. Here the last inequality follows from Lemmas 5 and 6 . Choose $\rho>0$ such that

$$
C_{1}\left\{\frac{C_{H}}{\delta^{d}}\left(\frac{|t-\tilde{t}|^{\frac{1}{2}} \vee|x-\tilde{x}|}{\delta}\right)^{\alpha}\right\} \frac{C_{0}}{d-\gamma} \rho^{d-\gamma}=2 \frac{C_{0} C_{1}}{\rho^{\gamma}}
$$

that is 


$$
\frac{1}{\rho}=\left\{\frac{C_{H}}{2(d-\gamma) \delta^{d}}\left(\frac{|t-\tilde{t}|^{\frac{1}{2}} \vee|x-\tilde{x}|}{\delta}\right)^{\alpha}\right\}^{1 / d} .
$$

Then

$$
H \leq 4 C_{0} C_{1}\left\{\frac{C_{H}}{2(d-\gamma) \delta^{d}}\left(\frac{|t-\tilde{t}|^{\frac{1}{2}} \vee|x-\tilde{x}|}{\delta}\right)^{\alpha}\right\}^{\gamma / d},
$$

which yields the claim.

Corollary 13 Under the same conditions for $\omega_{0}$ as in the previous section. Suppose the kernel $K=\left(K_{j}^{i}\right)$ satisfies the following condition:

$$
|K(x)| \leq C_{0} \frac{1}{|x|^{\gamma}} \quad \text { for all } x \neq 0
$$

where $0 \leq \gamma<d$ and $C_{0}>0$. Then there is a unique strong solution $X(x, t)$ to (2.24) for any $t \leq T_{L}$, such that

$$
b^{i}(x, t)=\int_{\mathbb{R}^{d}}\left(K_{j}^{i} \star \mathscr{L}(X(y, s))\right) \omega_{0}^{j}(y) d y
$$

$i=1, \cdots, d$, are bounded and Hölder continuous on any compact subset of $\mathbb{R}^{d} \times\left(0, T_{L}\right]$.

\section{From SDE to PDE}

In this section we recover the PDE from the SDE (2.24).

Theorem 14 Let $K$ and $\omega_{0}$ satisfy the assumptions in Sect. 4 . Let $\left(X(x, t), B_{t}\right)$ (where $x \in \mathbb{R}^{d}$ and $t \geq 0)$ be the unique weak solution of $\operatorname{SDE}(2.24)$ on a probability space $(\Omega, \mathcal{F}, \mathbb{P})$ for $t \in\left[0, T_{L}\right]$. Then for any $y \in \mathbb{R}^{d}$ and $t>0$, the distribution of $X(y, t)$ has a positive and continuous density denoted by $p(0, y, t, \cdot)$. Let $b(x, t)$ be defined by

$$
b^{i}(x, t)=\int_{\mathbb{R}^{d}}\left(K_{j}^{i} \star \mathscr{L}(X(y, s))\right) \omega_{0}^{j}(y) d y
$$

for $i=1, \ldots, d$, and

$$
\omega^{i}(x, t)=\int_{\mathbb{R}^{d}} p(0, y, t, x) \omega_{0}^{i}(y) d y
$$

for any $x$ and $t \in\left[0, T_{L}\right]$. Then the pair $(b, \omega)$ is the solution to the following non-local partial differential equation

$$
\frac{\partial}{\partial t} \omega^{i}+b^{j} \frac{\partial}{\partial x^{j}} \omega^{i}=\frac{1}{2} \Delta \omega^{i}-\frac{\partial b^{j}}{\partial x^{j}} \omega^{i}, \quad \omega^{i}(\cdot, 0)=\omega_{0}^{i}
$$

and

$$
b^{i}(x, t)=\int_{\mathbb{R}^{d}} K_{j}^{i}(x-y) \omega^{j}(y, t) d y
$$

for any $x$ any $t \in\left[0, T_{L}\right]$, where $i=1, \ldots, d$.

Proof According to our construction $b(x, t)$ is the unique bounded vector field such that $K \diamond b=b$, and $X(x, t)$ is the unique weak solution of the SDE

$$
d X(x, t)=b(X(x, t), t) d t+d B_{t}, \quad X(x, 0)=x .
$$


Thus $p(0, y, t, x)=p_{b}(0, y, t, x)$ is the transition probability density for the diffusion with its generator $L_{b}$, hence considering $p_{b}(0, y, t, x)$ as a function of $(t, x), p_{b}$ is the fundamental solution to the forward adjoint equation

$$
\left(\frac{\partial}{\partial t}-L_{b}^{\star}\right) p_{b}=0
$$

where $L_{b}^{\star}=\frac{1}{2} \Delta-b \cdot \nabla-\nabla \cdot b$. Hence, according to Lemma $1 \omega(x, t)$ given by $(6.1)$ is the solution to

$$
\left(\frac{\partial}{\partial t}-L_{b}^{\star}\right) \omega=0, \quad \omega(\cdot, 0)=\omega_{0}
$$

that is

$$
\frac{\partial}{\partial t} \omega+b \cdot \nabla \omega=\frac{1}{2} \Delta \omega-(\nabla \cdot b) \omega, \quad \omega(\cdot, 0)=\omega_{0} .
$$

Moreover,

$$
\begin{aligned}
b^{i}(x, t) & =\int_{\mathbb{R}^{d}}\left(K_{j}^{i} \star \mathscr{L}(X(y, s))\right) \omega_{0}^{j}(y) d y \\
& =\int_{\mathbb{R}^{d}}\left(\int K_{j}^{i}(x-z) p_{b}(0, y, t, z) d z\right) \omega_{0}^{j}(y) d y \\
& =\int_{\mathbb{R}^{d}} K_{j}^{i}(x-z) \omega^{j}(z, t) d z
\end{aligned}
$$

which completes the proof.

As an example we may apply this representation theorem to the Biot-Savart kernel $G(x)=$ $-\frac{x}{|x|^{3}}$ on $\mathbb{R}^{3}$ so that $K_{j}^{i}=\varepsilon^{i k j} G^{k}$. Then there is a unique weak solution to the following SDE

$$
d X(x, t)=\left(\left.\int_{\mathbb{R}^{3}} \mathbb{E}[G(z-X(y, t))]\right|_{z=X(x, t)} \times \omega_{0}(y) d y\right) d t+\sqrt{2 v} d B_{t}
$$

where $\omega_{0} \in L^{1}\left(\mathbb{R}^{3}\right) \cap L^{\infty}\left(\mathbb{R}^{3}\right)$ is the initial vorticity. In this case we define

$$
\omega(x, t)=\int_{\mathbb{R}^{3}} p(0, y, t, x) \omega_{0}(y) d y
$$

where $p(0, y, t, \cdot)$ is the probability density function of the law of $X(y, t)$ to $(6.4)$ and define

$$
\begin{aligned}
u^{i}(x, t) & =\int_{\mathbb{R}^{3}} K_{j}^{i}(x-z) \omega^{j}(z, t) d z \\
& =-\int_{\mathbb{R}^{3}} \varepsilon^{i k j} \frac{x^{k}-z^{k}}{|x-z|^{3}} \omega^{j}(z, t) d z .
\end{aligned}
$$

That is

$$
u(x, t)=-\int_{\mathbb{R}^{d}} \frac{x-z}{|x-z|^{3}} \times \omega(z, t) d z .
$$

By the previous theorem $(u, \omega)$ satisfies the following PDE

$$
\frac{\partial}{\partial t} \omega+u \cdot \nabla \omega=v \Delta \omega-(\nabla \cdot u) \omega, \quad \omega(\cdot, 0)=\omega_{0} .
$$


Moreover one can verify easily that

$$
\nabla \cdot u(x, t)=0 \quad \text { and } \Delta u(x, t)=-\nabla \times \omega(x, t)
$$

where the second equation follows from the Green formula which can be also written as

$$
\nabla \times(\nabla \times u-\omega)=0
$$

which yields that

$$
\omega=\nabla \times u-\nabla f
$$

for some scalar function $f$. If one also imposes a constraint: $\nabla \cdot \omega_{0}=0$ then $\omega=\nabla \times u$. In this case

$$
\frac{\partial}{\partial t} \omega+u \cdot \nabla \omega=v \Delta \omega, \quad \omega(\cdot, 0)=\omega_{0}
$$

and

$$
\omega=\nabla \times u .
$$

Hence $\left(X(x, t), B_{t}\right)$ is the probability representation of the solution to the above vorticity equation.

Acknowledgements Z. Qian would like to thank Professor Osada for sending him the paper [26] and a referee for bringing our attention to the paper [40].

Open Access This article is licensed under a Creative Commons Attribution 4.0 International License, which permits use, sharing, adaptation, distribution and reproduction in any medium or format, as long as you give appropriate credit to the original author(s) and the source, provide a link to the Creative Commons licence, and indicate if changes were made. The images or other third party material in this article are included in the article's Creative Commons licence, unless indicated otherwise in a credit line to the material. If material is not included in the article's Creative Commons licence and your intended use is not permitted by statutory regulation or exceeds the permitted use, you will need to obtain permission directly from the copyright holder. To view a copy of this licence, visit http://creativecommons.org/licenses/by/4.0/.

\section{References}

1. Aronson, D.G.: Bounds for the fundamental solution of a parabolic equation. Bull. Am. Math. Soc. 73(6), 890-896 (1967)

2. Aronson, D.G.: Non-negative solutions of linear parabolic equations. Annali della Scuola Normale Superiore di Pisa-Classe di Scienze 22(4), 607-694 (1968)

3. Aronson, D. G.: Uniqueness of positive weak solutions of second order parabolic equations. Ann. Polon. Math. 16(3), 285-303 (1965). http://eudml.org/doc/265270

4. Beale, J. T., Majda, A.: Vortex methods. I. Convergence in three dimensions. Math. Comput. 39(159), 1-27 (1982). https://doi.org/10.2307/2007617

5. Bauer, M., Meyer-Brandis, T., Proske, F.: Strong solutions of mean-field stochastic differential equations with irregular drift. Electron. J. Probab. 23(2018). https://doi.org/10.1214/18-EJP259

6. Buckdahn, R., Djehiche, B., Li, J., Peng, S.: Mean-field backward stochastic differential equations: a limit approach. Ann. Probab. 37(4), 1524-1565 (2009). https://www.jstor.org/stable/30244327

7. Buckdahn, R., Li, J., Peng, S., Rainer, C.: Mean-field stochastic differential equations and associated PDEs. Ann. Probab. 45(2), 824-878 (2017). https://doi.org/10.1214/15-AOP1076

8. Carmona, R., Delarue, F.: Probabilistic Theory of Mean Field Games with Applications I-II. Springer, New York (2018)

9. Chorin, A.J.: Numerical study of slightly viscous flow. J. Fluid Mech. 57(4), 785-796 (1973). https://doi. org/10.1017/S0022112073002016

10. Chorin, A. J.: Vorticity and Turbulence. Springer, New York (1994) 
11. Cottet, G. H.: A new approach for the analysis of vortex methods in two and three dimensions. Annales de l'Institut Henri Poincare (C) Non Linear Analysis 5(3), 227-285 (1988). http://www.numdam.org/item? id=AIHPC_1988_5_3_227_0

12. Cottet, G.H., Koumoutsakos, P.D.: Vortex Methods: Theory and Practice. Cambridge University Press, Cambridge (2000)

13. Fabes, E.B., Stroock, D.W.: A new proof of Moser's parabolic harnack inequality using the old ideas of Nash. Arch. Rational Mech. Anal. 96, 327-338 (1986). https://doi.org/10.1007/BF00251802

14. Friedman, A.: Partial Differential Equations of Parabolic Type. Prentice-Hall, Inc, New York (1964)

15. Funaki, T.: A certain class of diffusion processes associated with nonlinear parabolic equations. Zeitschrift $f^{\prime \prime}$ ur Wahrscheinlichkeitstheorie und Verwandte Gebiete, 67(3), 331-348 (1984). https://doi.org/10.1007/ BF00535008

16. Ikeda, N., Watanabe, S.: Stochastic Differential Equations and Diffusion Processes. North-Holland Publishing Company, Amsterdam (1989)

17. Karatzas, I., Shreve, S.: Brownian Motion and Stochastic Calculus. Springer, New York (1988)

18. Long, D.G.: Convergence of the random vortex method in two dimensions. J. Am. Math. Soc. 1(4), 779-804 (1988). https://doi.org/10.2307/1990992

19. Majda, A.J., Bertozzi, A.L.: Vorticity and Incompressible Flow. Cambridge University Press, Cambridge (2002)

20. Marchioro, C., Pulvirenti, M.: Hydrodynamics in two dimensions and vortex theory. Commun. Math. Phys. 84(4), 483-503 (1982). https://doi.org/10.1007/BF01209630

21. McKean, H.P., Jr.: A class of Markov processes associated with nonlinear parabolic equations. Proc. Natl. Acad. Sci. USA 56(6), 1907 (1966). https://doi.org/10.1073/pnas.56.6.1907

22. Méléard, S.: A trajectorial proof of the vortex method for the two-dimensional Navier-Stokes equation. Ann. Appl. Probab. 10(4), 1197-1211 (2000). https://doi.org/10.1214/aoap/1019487613

23. Nash, J.: Continuity of solutions of parabolic and elliptic equations. Am. J. Math. 80(4), 931-954 (1958). https://doi.org/10.2307/2372841

24. Norris, J., Stroock, D.: Estimates on the fundamental solution to heat flows with uniformly elliptic coefficients. Proc. Lond. Math. Soc. 62(3), 373-402 (1991)

25. Osada, H.: Propagation of chaos for the two dimensional Navier-Stokes equation. Proc. Jpn. Acad. Ser. A Math. Sci. 62(1), 8-11 (1986). https://doi.org/10.3792/pjaa.62.8

26. Osada, H.: Propagation of chaos for the two dimensional Navier-Stokes equation. In 'Taniguchi Symp. PMMP, Katata 1985', pp. 303-334 (1985)

27. Qian, Z., Russo, F., Zheng, W.: Comparison theorem and estimates for transition probability densities of diffusion processes. Probab. Theory Relat. Fields 127(3), 388-406 (2003). https://doi.org/10.1007/ s00440-003-0291-1

28. Qian, Z., Zheng, W.: A representation formula for transition probability densities of diffusions and applications. Stochast. Process. Appl. 111, 57-76 (2004)

29. de Raynal, P.C.: Strong well posedness of McKean-Vlasov stochastic differential equations with Hölder drift. Stochast. Process. Appl. 130(1), 79-107 (2020). https://doi.org/10.1016/j.spa.2019.01.006

30. Saffman, P.G.: Vortex Dynamics. Cambridge University Press, Cambridge (1995)

31. Stroock, D. W.: Diffusion semigroups corresponding to uniformly elliptic divergence form operators. Séminaire de Probabilités XXII , pp. 316-347. Springer, Berlin (1988). https://doi.org/10.1007/ BFb0084145

32. Stroock, D.W.: Partial Differential Equations for Probabilists. Cambridge University Press, Cambridge (2008)

33. Stroock, D. W., Varadhan, S. S.: Multidimensional Diffusion Processes. Springer, New York (1979)

34. Sznitman, A. S.: Topics in propagation of chaos. Ecole d'été de probabilités de Saint-Flour XIX-1989, pp. 165-251. Springer, Berlin (1991). https://doi.org/10.1007/BFb0085169

35. Talay, D., Tomašević, M.: A new McKean-Vlasov stochastic interpretation of the parabolic-parabolic Keller-Segel model: the one-dimensional case. Bernoulli 26(2), 1323-1353 (2020). https://doi.org/10. 3150/19-BEJ1158

36. Taylor, G.I.: Diffusion by continuous movements. Proc. Lond. Math. Soc. 2(1), 196-212 (1922). https:// doi.org/10.1112/plms/s2-20.1.196

37. Ting, L., Klein, R., Knio, O. M.: Vortex Dominated Flows: Analysis and Computation for Multiple Scale Phenomena. Springer, New York (2007)

38. Tomašević, M.: A new McKean-Vlasov stochastic interpretation of the parabolic-parabolic Keller-Segel model: The two-dimensional case. Ann. Appl. Probab. 31(1), 432-459 (2021). https://doi.org/10.1214/ 20-AAP1594

39. Veretennikov, A.J.: On strong solutions and explicit formulas for solutions of stochastic integral equations. Math. USSR-Sbornik 39(3), 387 (1981) 
40. Zhao, H.: On gradients of approximate travelling waves for generalised KPP equations. Proc. R. Soc. A Math. Phys. Eng. Sci. 127A, 423-439 (1997)

41. Zvonkin, A. K., Krylov, N. V.: Strong solutions of stochastic differential equations. Proceedings of the school and seminar on the theory of random processes (Druskininkai, 1974), Part II (Russian) (pp. 9-88) (1975) 